\title{
A case of treatment-induced neuropathy in an adolescent with type 1 diabetes
}

Niranjana Varadharaju, MBBS, MD ${ }^{1}$, Dhivyalakshmi Jeevarathnam, MD², Mahalakshmi Rajan, $\mathrm{MD}^{3}$, Vinoth Ponnurangam Nagarajan, $M D^{3}$, Saji James, $M^{3}$

${ }^{1}$ Sri Ramachandra Medical College, Chennai, India

${ }^{2}$ Division of Paediatric Endocrinology, Department of Pediatrics, Sri Ramachandra Medical College, Chennai, India

${ }^{3}$ Department of Pediatrics, Sri Ramachandra Medical College, Chennai, India

Received: 28 September, 2018 Revised: 22 November, 2018 Accepted: 22 December, 2018

Address for correspondence: Dhivyalakshmi Jeevarathnam, MD Department of Pediatrics, Division of Pediatric Endocrinology, G-block, 4th floor (link room), Sri Ramachandra Medical College and Hospital, Porur, Chennai-600116, India

Tel: +91-44-24330846

Fax: +91-44-24767008

E-mail: laksdhivya@gmail.com

https://orcid.org/0000-0003-1138$564 X$
Treatment-induced neuropathy (TIN) in diabetes is an acute and painful yet completely reversible small fiber neuropathy precipitated by a rapid improvement in glycemic control. TIN is rare in children. A 16-year-old girl developed symmetrical painful neuropathy of the foot, autonomic neuropathy, and retinopathy 5 weeks after the diagnosis of type 1 diabetes. All causative workups were negative except for a drop-in hemoglobin $A_{1 c}\left(H b A_{1 c}\right)$ from $17.4 \%$ to $7 \%$, which fit with a diagnosis of TIN. Following symptomatic management, her neuropathy and retinopathy completely resolved in 2 months. Currently, she is 18 years old and doing well ( $\left.\mathrm{HbA}_{1 c}, 7.4 \%\right)$ without any recurrence of TIN. TIN should be suspected in any child presenting with recent-onset type 1 diabetes and acute onset neuropathy. Our case represents an unreported scenario of the rapid progression in TIN. Awareness among clinicians about this rare but completely reversible condition is necessary to ensure proper management and adherence to glycemic control.

Keywords: Painful neuropathy, Autonomic neuropathy, Type 1 diabetes mellitus, Child, Glycemic control

\section{Introduction}

Acute painful diabetic neuropathy (APDN) is a rare peripheral neuropathy seen in type 1 as well as in type 2 diabetes. In APDN, neuropathic pain mostly occurs within 8 weeks of glycemic control in contrast to the rather insidious onset of diabetic distal polyneuropathy. Treatment-induced neuropathy (TIN) is a type of APDN. The criteria for TIN are acute onset of neuropathic pain and/or autonomic symptoms occurring within 8 weeks of documented glycemic control (a drop-in hemoglobin Alc [ $\left.\mathrm{HbA}_{1 \mathrm{c}}\right]$ by more than $2 \%$ over 3 months). There are many reports of TIN in adults with type 1 and type 2 diabetes, but not in children. TIN is observed with insulin therapy as well as treatment with oral hypoglycemic agents. ${ }^{1}$

\section{Case report}

A 16-year-old girl weighing $40 \mathrm{~kg}$ presented to the Emergency Department with a history of vomiting and excessive fatigability for the past week. She had a history of polyuria, polydipsia, and polyphagia for the past 1.5 years. She had taken some native medical treatment and reportedly experienced partial relief of her symptoms. Upon physical examination, she was conscious and alert and had tachycardia (heart rate, 120 beats/min) and tachypnea (respiratory rate, 20 breaths/min). Her blood pressure was $100 / 70 \mathrm{mmHg}$, and peripheral pulses were palpable. She was diagnosed with diabetic ketoacidosis (DKA) with a random blood glucose level of $560 \mathrm{mg} / \mathrm{dL}$, blood $\mathrm{pH}$ of 7.12 , and urine ketones $3+$. As per standard treatment protocol, she was treated with a normal saline bolus $(10 \mathrm{~mL} / \mathrm{kg}$ over 1 hour) followed by normal saline maintenance infused at $155 \mathrm{~mL} / \mathrm{kg} / \mathrm{hr}$ for 48 hours while accounting for $10 \%$ dehydration correction. An insulin infusion was started at $0.1 \mathrm{U} / \mathrm{kg} / \mathrm{hr}$. The DKA resolved by 
48 hours, and she was treated with regular (short-acting) and glargine insulin (regular insulin [Actrapid] was given at $12 \mathrm{U}$ - $16 \mathrm{U}$ - $12 \mathrm{U}$ subcutaneously half an hour before food intake and glargine was administered at 10 units subcutaneously at 9:00 PM). Her $\mathrm{HbA}_{\mathrm{lc}}$ was 17.4\%. C-peptide was $0.05 \mathrm{ng} / \mathrm{mL}$, and a glutamic acid decarboxylase antibody test was positive (14.8 $\mathrm{U} / \mathrm{mL}$; the normal range is $<10 \mathrm{U} / \mathrm{mL}$ ). She was screened for complications like retinopathy and nephropathy, which were found to be negative. Her thyroid profile (thyroid stimulating hormone, $3.2 \mu \mathrm{U} / \mathrm{mL}$; free thyroxine, $0.92 \mathrm{ng} / \mathrm{dL}$ ) was within normal limits. She was discharged a week after the diagnosis following symptomatic improvement, and her blood sugars ranged between $150-200 \mathrm{mg} / \mathrm{dL}$.

Five weeks following her diagnosis, the patient presented to the Outpatient Department with a painful burning sensation in both feet and soles for the past 10 days, which left her unable to walk and caused sleep disturbances. There was no change in her appetite or weight. Upon evaluation, her blood pressure was $100 / 70 \mathrm{mmHg}$, and she had normal superficial and deep tendon reflexes of the lower limbs. Her sensory system examination revealed no abnormalities for pain, temperature, vibration, position, or crude and fine touch sensations in both lower limbs. There were no motor or sensory deficits in either upper limb. She had vitamin D insufficiency $(15.7 \mathrm{ng} / \mathrm{mL})$ and a normal vitamin $B_{12}$ level $(240 \mathrm{pg} / \mathrm{mL})$. However, her pain had not subsided with nonsteroidal anti-inflammatory drugs along with vitamin $\mathrm{B}_{12}$ and cholecalciferol supplements. Her blood glucose records showed blood sugars around 100-150 mg/ $\mathrm{dL}$. Since the pain had not subsided after 2 weeks of outpatient treatment, she was admitted for diabetic peripheral neuropathy for further evaluation. At admission, she had normal motor reflexes of all 4 limbs. Her sensory system examination revealed reduced fine touch sensation in both feet and soles. There were no abnormalities in pain, temperature, vibration, and position sensations. There was hypertension (blood pressure, $150 / 100 \mathrm{mmHg}$ ) and resting tachycardia (122 beats/min). A hypertension work-up showed normal serum electrolytes and serum creatinine and an absence of microalbuminuria. Her computed tomography angiogram of the renal vessels, echocardiogram, vanillylmandelic acid, and metanephrine levels were normal. A vasculitis work-up (antinuclear antibodies, dsDNA, and antineutrophil cytoplasmic antibodies) was also negative. A pediatric cardiologist's opinion was obtained; the possibility of autonomic neuropathy was suggested, and antihypertensives (atenolol and nifedipine) were started.

A nerve conduction study revealed preserved compound muscle action potentials and normal distal latency and conduction velocities in both lower limb motor nerves (tibial and common peroneal nerve), whereas there was a significant reduction in sensory nerve action potentials with mildly prolonged distal latency and mildly reduced conduction velocities in both superficial peroneal and sural nerves in the lower limbs suggestive of severe bilateral lower limb axonal predominant sensory polyneuropathy. Upper limb nerve conduction studies were normal.
During her course of treatment, the patient complained of the sudden onset of blurring of her vision, and her ophthalmologic examination revealed features suggestive of diabetic and hypertensive retinopathy. Fundus fluorescein angiography showed areas of capillary nonperfusion of both eyes with macular ischemia and cystoid macular edema suggestive of diabetic and hypertensive retinopathy. She was started on amitriptyline, pregabalin, and gabapentin for peripheral neuropathy.

Aided by electronic discussions in an International Society for Pediatric and Adolescent Diabetes (ISPAD) forum along with a thorough literature search, the cause of the neuropathy was diagnosed to be TIN. The patient's $\mathrm{HbA}_{1 \mathrm{c}}$ had dropped from $17.4 \%$ to $7 \%$ in 2 months, which fit the diagnosis of TIN. ${ }^{1)}$

Following the diagnosis of TIN, the patient's glycemic targets were slightly relaxed. She was discharged with antihypertensives and peripheral neuropathy medications on withdrawing doses. At the follow-up visit around 2 months later, the neuropathy (peripheral and autonomic) and retinopathy had both gradually resolved. All medications were stopped, and her $\mathrm{HbA}$ 1c was 8.6\%. Her glycemic control targets were gradually changed back to the recommended levels according to the ISPAD 2014 guidelines. ${ }^{2)}$ Currently, she is 18 years old, and her $\mathrm{HbA}_{1 \mathrm{c}}$ was $7.4 \%$ without any recurrence of TIN. She is currently on regular insulin (Actrapid $10 \mathrm{U}$ - $16 \mathrm{U}$ - $12 \mathrm{U}$ subcutaneously half an hour before food intake and glargine insulin 11 units subcutaneously at 9:00 PM).

\section{Discussion}

APDN is a rare peripheral neuropathy seen in type 1 as well as in type 2 diabetes. It was initially reported in 1933 by Caravati ${ }^{3}$ as insulin neuritis. The incidence of APDN is not known or is likely grossly underestimated and is probably less than 1 in 1,000 diabetic patients. APDN can manifest as either treatmentinduced neuropathy (TIN) and diabetic neuropathic cachexia (DNC). ${ }^{1,3)}$

In TIN, neuropathic pain and/or autonomic symptoms typically occur within 8 weeks of documented glycemic control (a drop-in $\mathrm{HbA}_{1 \mathrm{c}} \geq 2 \%$ over 3 months). Several weeks after the onset, more classical length-dependent axonal and demyelinating sensory and motor neuropathy can occur. ${ }^{1,4)} \mathrm{A}$ retrospective study by Gibbons and Freeman ${ }^{4)}$ observed the incidence of TIN to be $10.9 \%$ among 954 patients referred for diabetic neuropathy. TIN can occur with insulin and/or oral hypoglycemic agent therapy. To the best of our knowledge, there are only 7 case reports of TIN in children with diabetes, which highlights the rarity of this clinical entity. ${ }^{5-10)}$ TIN completely resolves in about 12-24 months; however, in our patient, the early onset of neuropathy with a quick progression to autonomic neuropathy and retinopathy and its resolution within weeks was quite unusual compared to previously published reports. The proposed diagnostic criteria for TIN are listed in Table 1.

TIN is caused due to epineural arteriovenous shunting triggered by rapid glycemic control that precipitates endoneural 
Table 1. Proposed diagnostic criteria for treatment-induced neuropathy*

\begin{tabular}{|c|c|}
\hline & Treatment-induced neuropathy \\
\hline History & $\begin{array}{l}\text { Improved glycemic control (type } 1 \text { or type } 2 \text { diabetes) with insulin or oral hypoglycemic agents } \\
\text { And } \\
\text { - Onset of neuropathic pain within } 6 \text { months of treatment } \\
\text { - Autonomic symptoms } \\
\text { - History of eating disorder in type } 1 \text { diabetes }\end{array}$ \\
\hline Clinical examination & Painful paresthesia and symptoms of autonomic neuropathy \\
\hline Nerve conduction studies & Sensorimotor polyneuropathy \\
\hline Autonomic testing & Parasympathetic and sympathetic dysfunction \\
\hline Laboratory tests & $\begin{array}{l}\text { Baseline } \mathrm{HbA}_{1 c}>10 \% \\
\text { And } \\
\mathrm{Hb}_{1 \mathrm{c}} \text { after treatment }<9 \% \text { or drop of } \mathrm{Hb}_{1 \mathrm{c}}>2 \% \text { within } 3 \text { months } \\
\text { And } \\
\text { Normal values of TSH, ANA, ESR, vitamin } \mathrm{B}_{12} \text {, vitamin } \mathrm{B}_{1} \text {, Serum protein electrophoresis and light chain analysis }\end{array}$ \\
\hline Differential diagnosis excluded & $\begin{array}{l}\text { Other polyneuropathies (Guillain-Barré variant, acute steroid-responsive small fibre sensory neuropathy and non- } \\
\text { systemic nerve vasculitis) }\end{array}$ \\
\hline
\end{tabular}

$\mathrm{HbA}_{1 c}$, glycated hemoglobin; TSH, thyroid stimulating hormone; ANA, antinuclear antibody; ESR, erythrocyte sedimentation rate.

"Modified from Tran C, et al. Swiss Med Wkly 2015;145:w14131.")

hypoxia in small nerve fibers (the steal effect). DNC is a painful sensorimotor polyneuropathy with autonomic dysfunction that follows a recent diabetes onset, but a history of an eating disorder along with profound weight loss (>10\% of body weight) is always present. ${ }^{1,11,12)}$

The most common differential diagnoses for TIN as well as the required laboratory tests are shown in Table 1. The gold standard test for diagnosing small fiber neuropathy is skin biopsy with intraepidermal nerve fiber density (IENFD) determination. ${ }^{13,14)}$ Due to logistic reasons, we could not carry out protein electrophoresis, light chain serum levels, and IENFD, but all the other investigations were normal for our patient.

Treatment options involve good glycemic control, foot care, and pharmacological management. It may be safe to achieve glycemic control slowly and gradually if there is no risk of hyperglycemic emergencies in these patients. ${ }^{1)}$ According to the ISPAD 2014 guidelines for optimal blood sugar control, an $\mathrm{HbA}_{\mathrm{lc}}<7.5 \%$ is recommended as soon as it can be achieved. ${ }^{2)}$ However, a study by Gibbons and Freeman ${ }^{4)}$ has shown that a decrease in $\mathrm{HbA}_{1 \mathrm{c}}$ by $2 \%-3 \%$ over 3 months has a $20 \%$ absolute risk, while a fall in $\mathrm{HbA}_{1 \mathrm{c}}$ by $>4 \%$ over 3 months exceeds $80 \%$ of the absolute risk for developing TIN. ${ }^{4)}$ Therefore, in the recommended setting of early achievement of good glycemic control, awareness about TIN and its management among clinicians is necessary to ensure adequate treatment.

Pharmacological treatment for neuropathic pain includes anticonvulsants (pregabalin, gabapentin), antidepressants (duloxetine, amitriptyline), and opioids (tramadol, tapentadol). Pharmacological agents are started with a single drug (mostly pregabalin) in incremental doses until the maximum dose is reached. If neuropathic pain persists, switching to another drug or combined therapy (with antidepressants) can be tried. If the pain still persists, opioids can be added. ${ }^{15)}$

In conclusion, clinicians should suspect TIN in any diabetic child, especially with neuropathy presenting too early in the course along with good glycemic control. Our case represents the unreported scenario of rapid progression to TIN. Awareness among clinicians about this rare and completely reversible condition is necessary to ensure proper treatment and adherence to glycemic control, especially in the recommended setting of the rapid achievement of glycemic control ( $\mathrm{HbA}_{1 \mathrm{c}}$ $<8 \%$ ). Instead of rapid improvement in glycemic control, a slow and gradual achievement of glycemic control may be a better option for these patients.

\section{Ethical statement}

As per the Institution's ethics committee policy, Institutional Review Board approval is not necessary for case reports. Informed consent has been obtained from the patient for publication purposes.

\section{Conflict of interest}

No potential conflict of interest relevant to this article was reported.

\section{Acknowledgments}

We thank Dr. Ragnar Hanas, past president of ISPAD, for the excellent guidance provided in the diagnosis and management of this patient.

\section{References}

1. Tran C, Philippe J, Ochsner F, Kuntzer T, Truffert A. Acute painful diabetic neuropathy: an uncommon, remittent type of acute distal small fibre neuropathy. Swiss Med Wkly 2015;145:w14131. 
2. Rewers MJ, Pillay K, de Beaufort C, Craig ME, Hanas R, Acerini CL, et al. ISPAD Clinical Practice Consensus Guidelines 2014. Assessment and monitoring of glycemic control in children and adolescents with diabetes. Pediatr Diabetes 2014;15 Suppl 20:102-14.

3. Caravati CM. Insulin neuritis: a case report. Va Med Mon 1933;59:745-6.

4. Gibbons CH, Freeman R. Treatment-induced neuropathy of diabetes: an acute, iatrogenic complication of diabetes. Brain 2015;138(Pt 1):43-52.

5. Archer AG, Watkins PJ, Thomas PK, Sharma AK, Payan J. The natural history of acute painful neuropathy in diabetes mellitus. J Neurol Neurosurg Psychiatry 1983;46:491-9.

6. Wilson JL, Sokol DK, Smith LH, Snook RJ, Waguespack SG, Kincaid JC. Acute painful neuropathy (insulin neuritis) in a boy following rapid glycemic control for type 1 diabetes mellitus. J Child Neurol 2003;18:365-7.

7. Llewelyn JG, Thomas PK, Fonseca V, King RH, Dandona P. Acute painful diabetic neuropathy precipitated by strict glycaemic control. Acta Neuropathol 1986;72:157-63.

8. Mathews C, Rayman G, Buck J, Wadham C, Perkins E. Acute treatment induced diabetic neuropathy in a 15 -yearold boy. Proceedings of the 45th Meeting of the British Society for Paediatric Endocrinology and Diabetes; 2017 Nov 22-24; Newcastle. Bristol: British Society for Paediatric Endocrinology and Diabetes, 1979.

9. Dayal D, Jayaraman D, Sankhyan N, Singhi P. Acute painful neuropathy in a girl with type 1 diabetes: long term follow- up. J Clin Diagn Res 2016;10:SD01-2.

10. Rangel MA, Baptista C, Santos F, Real MV, Campos RA, Leite AL. Acute mononeuropathy in a child with newly diagnosed type 1 diabetes mellitus. J Pediatr Endocrinol Metab 2015;28:341-4.

11. Tesfaye S, Malik R, Harris N, Jakubowski JJ, Mody C, Rennie IG, et al. Arterio-venous shunting and proliferating new vessels in acute painful neuropathy of rapid glycaemic control (insulin neuritis). Diabetologia 1996;39:329-35.

12. Leow MK, Wyckoff J. Under-recognised paradox of neuropathy from rapid glycaemic control. Postgrad Med J 2005;81:103-7.

13. England JD, Gronseth GS, Franklin G, Carter GT, Kinsella LJ, Cohen JA, et al. Practice Parameter: evaluation of distal symmetric polyneuropathy: role of laboratory and genetic testing (an evidence-based review). Report of the American Academy of Neurology, American Association of Neuromuscular and Electrodiagnostic Medicine, and American Academy of Physical Medicine and Rehabilitation. Neurology 2009;72:185-92.

14. Devigili G, Tugnoli V, Penza P, Camozzi F, Lombardi R, Melli G, et al. The diagnostic criteria for small fibre neuropathy: from symptoms to neuropathology. Brain 2008;131(Pt 7):1912-25.

15. Pop-Busui R, Boulton AJ, Feldman EL, Bril V, Freeman R, Malik RA, et al. Diabetic neuropathy: a position statement by the American Diabetes Association. Diabetes Care 2017;40:136-54. 